\title{
i4mC-Deep: An Intelligent Predictor of N4-Methylcytosine Sites Using a Deep Learning Approach with Chemical Properties
}

\author{
Waleed Alam ${ }^{1}\left(\mathbb{D}\right.$, Hilal Tayara ${ }^{2, *} \mathbb{D}$ and Kil To Chong $1,3, * \mathbb{D}$ \\ 1 Department of Electronics and Information Engineering, Jeonbuk National University, Jeonju 54896, Korea; \\ waleedtkr@jbnu.ac.kr \\ 2 School of International Engineering and Science, Jeonbuk National University, Jeonju 54896, Korea \\ 3 Advanced Electronics and Information Research Center, Jeonbuk National University, Jeonju 54896, Korea \\ * Correspondence: hilaltayara@jbnu.ac.kr (H.T.); kitchong@jbnu.ac.kr (K.T.C.)
}

Citation: Alam, W.; Tayara, H.;

Chong, K.T. i4mC-Deep:

An Intelligent Predictor of

N4-Methylcytosine Sites Using a Deep Learning Approach with

Chemical Properties. Genes 2021, 12,

1117. https://doi.org/10.3390/

genes12081117

Academic Editor: Denis Bauer

Received: 7 June 2021

Accepted: 16 July 2021

Published: 23 July 2021

Publisher's Note: MDPI stays neutral with regard to jurisdictional claims in published maps and institutional affiliations.

Copyright: (c) 2021 by the authors. Licensee MDPI, Basel, Switzerland. This article is an open access article distributed under the terms and conditions of the Creative Commons Attribution (CC BY) license (https:// creativecommons.org/licenses/by/ $4.0 /)$.

\begin{abstract}
DNA is subject to epigenetic modification by the molecule N4-methylcytosine (4mC). N4-methylcytosine plays a crucial role in DNA repair and replication, protects host DNA from degradation, and regulates DNA expression. However, though current experimental techniques can identify $4 \mathrm{mC}$ sites, such techniques are expensive and laborious. Therefore, computational tools that can predict $4 \mathrm{mC}$ sites would be very useful for understanding the biological mechanism of this vital type of DNA modification. Conventional machine-learning-based methods rely on hand-crafted features, but the new method saves time and computational cost by making use of learned features instead. In this study, we propose i4mC-Deep, an intelligent predictor based on a convolutional neural network (CNN) that predicts $4 \mathrm{mC}$ modification sites in DNA samples. The CNN is capable of automatically extracting important features from input samples during training. Nucleotide chemical properties and nucleotide density, which together represent a DNA sequence, act as CNN input data. The outcome of the proposed method outperforms several state-of-the-art predictors. When i4mC-Deep was used to analyze G. subterruneus DNA, the accuracy of the results was improved by $3.9 \%$ and MCC increased by $10.5 \%$ compared to a conventional predictor.
\end{abstract}

Keywords: DNA methylation; regulate expression; CNN; deep learning

\section{Introduction}

In DNA modification, methylation is a fundamental epigenetic tag that plays a major role in biological processes such as genomic imprinting, preservation of chromosomal stability, X-chromosome inactivation, cell cycle progression, and regulation of gene expression [1,2]. Cytosine methylation has been widely studied in both eukaryotic and prokaryotic genomes, where it creates bases such as 5-methylcytosine (5mC), 3-methylcytosine, and N4-methylcytosine [3,4]. 5mC is produced by adding a methyl group via DNA methyltransferase (DNMT) to the C5 position of cytosine, whereas 3methylcytosine accrues due to the action of environmental alkylation agents $[3,5] .5 \mathrm{mC}$ is known to play a significant role in various biological functions [6,7] and is associated with diabetes, cancer, and neurological diseases [8-10]. In bacterial DNA, methylated DNA nucleobase $4 \mathrm{mC}$ is commonly explored, while the exact mechanisms and biological functions of $4 \mathrm{mC}$ modification sites are still limited [10].

The $4 \mathrm{mC}$ is relatively less investigated as compared to $5 \mathrm{mC}$, and it has several roles such as correcting and controlling the DNA replication, gene expression levels, and cell cycle $[2,11]$. There are various experimental techniques for the identification of epigenetic cytosine nucleobases $(4 \mathrm{mC})$, which are namely, whole-genome bisulfite sequencing, reduced-representation bisulfite sequencing, mass spectrometry, transcription-activatorlike effectors (TALEs) and single-molecule real-time sequencing (SMRT) [12-14]. Although 
these experimental techniques are sufficient for the identification of $4 \mathrm{mC}$ sites, they are expensive and laborious. Therefore, an efficient computational algorithm for the prediction of $4 \mathrm{mC}$ modification sites in large-scale genomic sequences would be greatly beneficial to the field. In the past decade, deep learning methods have achieved a remarkable performance in various fields such as image recognition [15-17], speech recognition [18], natural language processing [19] and bioinformatics [20-24].

Recently, several computational tools have been developed for the identification of $4 \mathrm{mc}$ sites, including iDNA4mC [25], 4mCPred [26], 4mCPred-SVM [27] and SOMM4mC [28]. All of these tools are based on machine learning techniques with hand-crafted features. iDNA4mC uses a support vector machine (SVM) with nucleotide chemical properties and nucleotide frequency as a feature vector for the detection of $4 \mathrm{mC}$ sites. $4 \mathrm{mCPred}$ and $4 \mathrm{mCPred}-\mathrm{SVM}$ also use an SVM but have different mechanisms of feature representation. $4 \mathrm{mCPred}$ relies on two feature-encoding techniques, position-specific trinucleotide propensity (PSTNP) and electron-ion interaction pseudopotential EIIPs of trinucleotides, to encode the DNA sample as discrete value vectors. 4mCPredSVM applies four types of features for a combinatorial approach to $4 \mathrm{mC}$ site prediction, namely, K-mer dinucleotide frequency, mono-nucleotide binary encoding, dinucleotide binary encoding, and local position-specific dinucleotide frequency. SOMM4mC applies classical first and secondorder Markov models to predict the $4 \mathrm{mC}$ epigenetic modification sites and shows better performance than the other previously mentioned tools. Furthermore, 4mCCNN [29] and DeepTorrent [30] are based on deep learning techniques. 4mCCNN utilizes one-hot encoding for data representation and convolution neural networks. DeepTorrent uses four type feature extraction techniques with convolution and LSTM layers. The previous deep learning model used complex architecture, which increases parameter and computational costs. Therefore, we need to design a more efficient model for $4 \mathrm{mC}$ site identification.

In this study, we employ a convolutional neural network (CNN) to develop an accurate and efficient computational tool. The $\mathrm{CNN}$ is based on several layers, including a convolutional layer, batch normalization layer, flatten layer, dropout layer, and dense layer. The convolutional layer is used to automatically extract important features from an encoded DNA sequence. We apply the nucleotide chemical properties (NCP) and nucleotide density (ND) methods to encode the input DNA sequences [25,31,32]. Moreover, we use the batch normalization and dropout layers to control overfitting. Finally, we utilize the dense layer with sigmoid activation to classify the DNA sequence as either a $4 \mathrm{mC}$ site or a non- $4 \mathrm{mC}$ site. We apply the 10 -fold cross-validation technique with standard evaluation metrics in the field of bioinformatics [33-35] to evaluate i4mC-Deep. The outcomes of i4mC-Deep are superior to those of previous tools. The architecture of the i4mC-Deep has been illustrated in Figure 1. Finally, we have developed a free online web server to facilitate research in academia and industry, which is available at http:/ /nsclbio.jbnu.ac.kr/tools/i4mC-Deep/, accessed on 15 July 2021, and we have provided the source code at: https:/ / github.com/waleed551/i4mC-Deep, accessed on 15 July 2021 . 


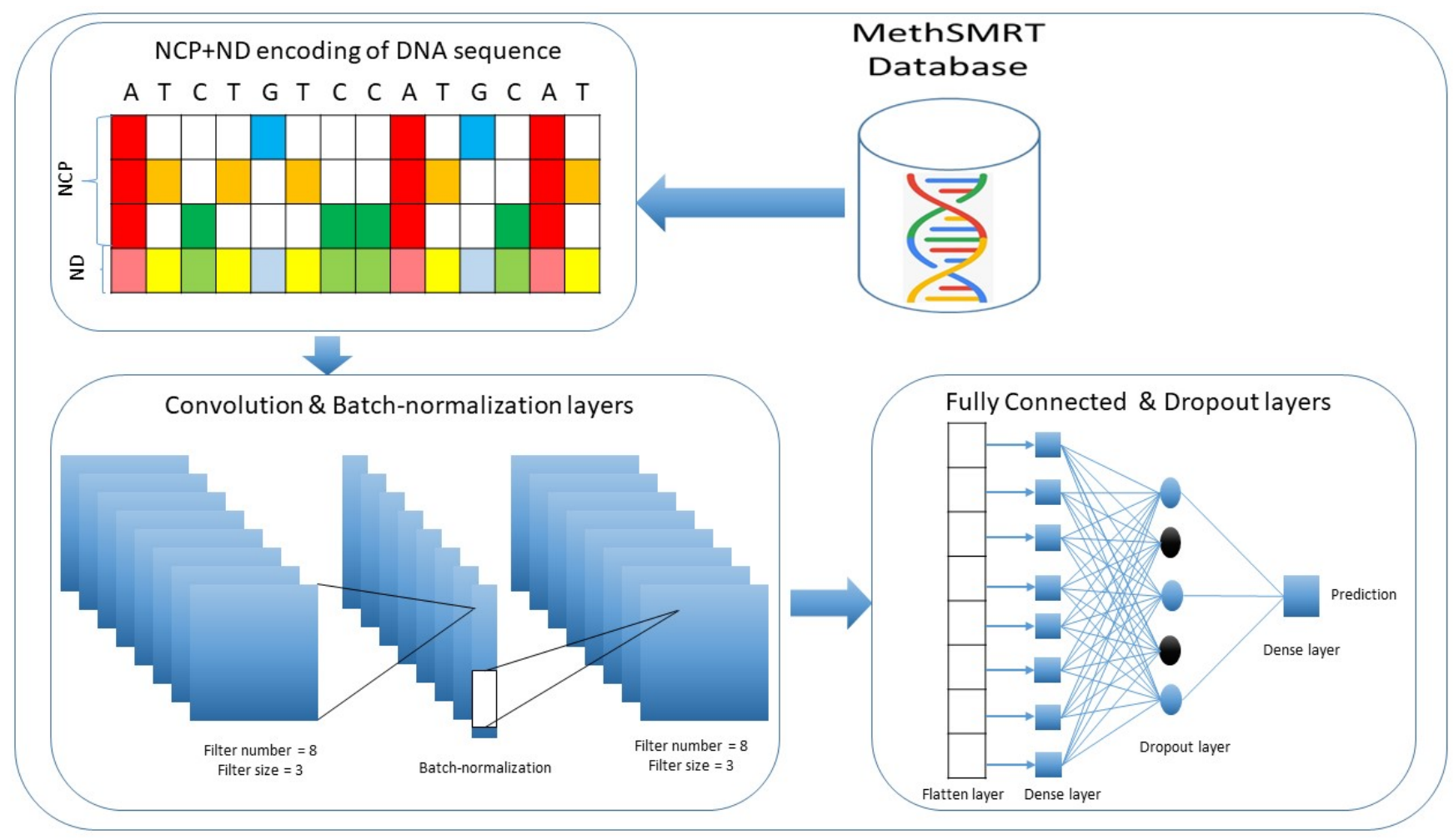

Figure 1. Demonstration of the data-flow and architecture of the proposed model.

\section{Materials and Methods}

This section includes the benchmark datasets, proposed model and evaluation measures.

\subsection{Benchmark Dataset}

The dataset plays a very important role in the development of an efficient and reliable computational tool. We utilized data from six different species of prokaryotes and eukaryotes, Caenorhabditis elegans, Drosophila melanogaster, Arabidopsis thaliana, Escherichia coli, Geoalkalibacter subterraneus, and Geobacter pickeringii. The datasets were constructed by [25] using the MethSMRT database [36]. The benchmark datasets contain 1554, 1769, $1978,388,906$, and 569 positive and negative samples, respectively. Each sequence in the six datasets has a centrally located cytosine (C), with a length of $41 \mathrm{nt}$. The summary of six species benchmark datasets is shown in Table 1.

Table 1. The summary of six species benchmark datasets.

\begin{tabular}{cccc}
\hline Species & \multicolumn{2}{c}{ Sequences } & Total \\
\hline \multirow{2}{*}{ C. elegans } & Positive & 1554 & 3108 \\
& Negative & 1554 & 3538 \\
D. melanogaster & Positive & 1769 & \\
& Negative & 1769 & 3956 \\
A. thaliana & Positive & 1978 & \\
& Negative & 1978 & 776 \\
E. coli & Positive & 388 & 1812 \\
\multirow{2}{*}{ G. subterraneus } & Negative & 388 & 1138 \\
G. pickeringii & Positive & 906 & \\
\hline
\end{tabular}




\subsection{Deep Learning Approach}

In this study, we used a convolutional neural network (CNN) to predict $4 \mathrm{mC}$ modification sites from DNA samples. The $\mathrm{CNN}$ is capable of automatically extracting important features from the input samples during training. The CNN input of DNA sequences is encoded by nucleotide chemical properties (NCP) and nucleotide density (ND). Each input DNA sequence has four different chemical properties that are derived from three groups based on the presence of hydrogen bonds, functional groups, and ring structures. In detail, during the formation of secondary structures, $\mathrm{A}$ and $\mathrm{T}$ form weak hydrogen bonds, whereas $C$ and $G$ form strong bonds; $G$ and $T$ contain a keto group, while $A$ and $C$ contain an amino group; and $\mathrm{C}$ and $\mathrm{T}$ have structures with only one ring, whereas $\mathrm{A}$ and $\mathrm{G}$ have two ring structures. Accordingly, the chemical properties of the four nucleotides can be represented in three coordinates $(x, y$, and $z)$, and each coordinate can be assigned a value of 0 or 1 . Thus, the four nucleotides that make up a DNA sequence can be represented in the Cartesian coordinate system. The resultant coordinates for A, C, G, and T are $(1,1,1),(0,0,1),(1,0,0)$ and $(0,1,0)$, respectively. The nucleotide density contains information on the frequency of each nucleotide in a given DNA sequence. Thus, we integrate NCP and ND into a 4-channel feature vector. The CNN has steps, or layers, including the convolution layers, activation layers, normalization layers, flatten layers, dropout layers, and fully connected layers. Several hyper-parameters are tuned during training, such as filter size, kernel size, strides, and dropout probability. The best hyper-parameter has been selected based on the validation loss. The gird search range of hyper-parameters is shown in Table 2. The optimal hyper-parameters for convolution layers are 2, the filter size is 8 for both layers, padding is the "same" for both layers, kernel size is 3 for both layers, and the dropout probability is 0.3 . The mathematical representation of these layers is as follows:

$$
\begin{gathered}
\operatorname{Conv}(S)_{i j}=\operatorname{ReLU}\left(\sum_{s=0}^{Z-1} \sum_{n=0}^{I-1} W_{s n}^{k} S_{j+s, n}\right) \\
\mathrm{f}=w_{d+1} \sum_{k=1}^{d} m_{k} w_{k} z_{k} \\
\operatorname{ReLU}(x)= \begin{cases}x & \text { if } x>0 \\
0 & \text { if } x \leq 0\end{cases} \\
\operatorname{Sigmoid}(x)=\frac{1}{1+e^{-x}}
\end{gathered}
$$

We adopted several evaluation measures, namely, sensitivity, specificity, accuracy, and Mathew correlation coefficient (MCC), to enable a fair evaluation of the proposed tools. Both convolution layers and one dense layer are followed by a nonlinear function rectified linear unit (ReLU), while the last dense layer is followed by a sigmoid activation function that classifies the given DNA sequence as a $4 \mathrm{mC}$ or non- $4 \mathrm{mC}$ site. The sigmoid activation function scales the output into the range [0,1]. Moreover, we applied 12 regularization and dropout regularization to avoid overfitting from the network. The proposed model has been optimized using Adam with a learning rate of 0.001 . The best batch size for the proposed model is 32 , and the ideal number of epochs is 200 with early stopping. This bioinformatics tool is implemented in Python using the Keras framework. 
Table 2. The ranges of the tuned hyper-parameters.

\begin{tabular}{lc}
\hline Hyper-Parameters & Range \\
\hline Filters of Conv1D & {$[8,16,32]$} \\
Conv1D kernel size & {$[3,5,7]$} \\
Conv1D Strides & {$[2,3]$} \\
Dropout & {$[0.2,0.3,0.4,0.5]$} \\
Dense layer units & {$[8,16,32]$} \\
\hline
\end{tabular}

\subsection{Evaluation Measures}

In this study, we applied four standard measures that are widely used in binary classification tasks to evaluate the performance of bioinformatics tools [37-43], namely, accuracy (ACC), sensitivity (SN), specificity (SP), and Matthew correlation coefficient (MCC). Mathematically, these measures are expressed as follows:

$$
\begin{gathered}
\mathrm{ACC}=1-\left(\frac{N_{-}^{+}+N_{+}^{-}}{N^{+}+N^{-}}\right) \\
\mathrm{SN}=1-\left(\frac{N_{-}^{+}}{N^{+}}\right) \\
\mathrm{SP}=1-\left(\frac{N_{+}^{-}}{N^{-}}\right) \\
\mathrm{MCC}=\frac{1-\left(\frac{N_{-}^{+}+N_{+}^{-}}{N^{+}+N^{-}}\right)}{\sqrt{\left(1+\frac{N_{+}^{-}-N_{-}^{+}}{N^{+}}\right)\left(1+\frac{N_{-}^{+}-N_{+}^{-}}{N^{-}}\right)}}
\end{gathered}
$$

where $\mathrm{N}^{+}$represents methylcytosine sites, $\mathrm{N}^{-}$represents nonmethylcytosine sites, $N_{+}^{-}$represents methylcytosine sites that are incorrectly identified as non-methylcytosine sites, and $\mathrm{N}_{-}^{+}$ shows the number of non-methylcytosine sites that are predicted to be methylcytosine sties.

\section{Result and Discussion}

\subsection{Comparison with Other State-of-the-Art Tools}

Here, we compare the performance of the proposed method with other state-of-the-art tools, including iDNA4mC [25], 4mCPred [26], 4mCPred-SVM ([27]), and SOMM4mC ([28]). Table 3 and Figure 2 demonstrate the performance according to the four basic evaluation metrics of the proposed method and existing methods. Figure 3 shows the receiver operation characteristic curve (ROC) of six species along with standard deviation errors in ten folds. As done in previous studies, we also utilize 10-fold cross-validation and similar measurement parameters to enable a fair comparative evaluation. The results show that the accuracy of the proposed method is superior to that of existing state-of-the-art methods for all benchmark datasets. In detail, $4 \mathrm{mC}$-Deep improved the classification of the C. elegans benchmark dataset by $1.0 \%$ accuracy, $3.5 \%$ sensitivity, and 3.1\% MCC. In D. melanogaster, accuracy was improved by $2.1 \%$, specificity by $0.6 \%$, sensitivity by $3.6 \%$, and MCC by $6.7 \%$. In $A$. thaliana, accuracy was improved by $2.9 \%$, sensitivity by $7.1 \%$, and MCC by $8.4 \%$. In $E$. coli, 4mC-Deep improved accuracy, sensitivity, and MCC by $0.8 \%, 1.9 \%$, and $0.1 \%$ respectively. All measurement parameters were improved in G. subterraneus: 3.9\% improvement in accuracy, $3.8 \%$ for specificity, $4.0 \%$ for sensitivity, and 10.5\% for MCC. In G. pinckeringii, classification was improved by $2.3 \%, 2.7 \%, 2.0 \%$, and $8.3 \%$ for accuracy, sensitivity, specificity, and MCC, respectively. As we notice, the specificity of the previous tools is higher than the proposed tool. Specificity demonstrates the correct detection of the true-negative classes, while sensitivity demonstrates the correct detection of the true-positive classes. Accordingly, the specificity and sensitivity are both highly required for better performance of the models. If we look at the performance of the previous method, the variance between the sensitivity and specificity is higher, which decreases the model accuracy and MCC. 
On the other hand, the performance of the proposed tool is much higher as compared with existing tools because the variance is less between sensitivity and specificity. Therefore, it is evident that the outcomes of our proposed 4mC-Deep tool outperformed all existing tools on six species benchmark datasets.

Table 3. The performance comparison between the i4mC-Deep and the existing computational tools for $4 \mathrm{mC}$ sites.

\begin{tabular}{|c|c|c|c|c|c|}
\hline Datasets & Methods & $\mathrm{ACC}$ & SN & SP & MCC \\
\hline \multirow{7}{*}{ C. elegans } & iDNA4mC & 0.786 & 0.797 & 0.775 & 0.572 \\
\hline & $4 \mathrm{mCPred}$ & 0.826 & 0.825 & 0.826 & 0.652 \\
\hline & 4mCPred-SVM & 0.815 & 0.824 & 0.807 & 0.631 \\
\hline & $4 \mathrm{mCCNN}$ & 0.842 & 0.894 & 0.825 & 0.694 \\
\hline & DeepTorrent & 0.858 & 0.810 & 0.906 & 0.719 \\
\hline & SOMM4mC & 0.876 & 0.839 & 0.913 & 0.743 \\
\hline & i4mC-Deep & 0.886 & 0.874 & 0.898 & 0.774 \\
\hline \multirow{7}{*}{ D. melanogaster } & iDNA4mC & 0.812 & 0.833 & 0.791 & 0.625 \\
\hline & $4 \mathrm{mCPred}$ & 0.822 & 0.824 & 0.821 & 0.646 \\
\hline & 4mCPred-SVM & 0.830 & 0.838 & 0.822 & 0.661 \\
\hline & $4 \mathrm{mCCNN}$ & 0.853 & 0.864 & 0.853 & 0.686 \\
\hline & DeepTorrent & 0.861 & 0.834 & 0.889 & 0.724 \\
\hline & SOMM4mC & 0.874 & 0.862 & 0.886 & 0.724 \\
\hline & i4mC-Deep & 0.895 & 0.898 & 0.892 & 0.791 \\
\hline \multirow{7}{*}{ A. thaliana } & iDNA4mC & 0.760 & 0.757 & 0.762 & 0.519 \\
\hline & $4 \mathrm{mCPred}$ & 0.768 & 0.755 & 0.780 & 0.536 \\
\hline & 4mCPred-SVM & 0.787 & 0.778 & 0.796 & 0.573 \\
\hline & $4 \mathrm{mCCNN}$ & 0.797 & 0.803 & 0.792 & 0.621 \\
\hline & DeepTorrent & 0.803 & 0.703 & 0.903 & 0.620 \\
\hline & SOMM4mC & 0.836 & 0.800 & 0.872 & 0.647 \\
\hline & i4mC-Deep & 0.865 & 0.871 & 0.861 & 0.731 \\
\hline \multirow{7}{*}{ E. coli } & iDNA4mC & 0.799 & 0.820 & 0.778 & 0.598 \\
\hline & $4 \mathrm{mCPred}$ & 0.826 & 0.819 & 0.832 & 0.655 \\
\hline & 4mCPred-SVM & 0.833 & 0.858 & 0.807 & 0.666 \\
\hline & $4 \mathrm{mCCNN}$ & 0.859 & 0.881 & 0.788 & 0.687 \\
\hline & DeepTorrent & 0.873 & 0.891 & 0.855 & 0.747 \\
\hline & SOMM4mC & 0.918 & 0.903 & 0.934 & 0.853 \\
\hline & i4mC-Deep & 0.926 & 0.930 & 0.922 & 0.854 \\
\hline \multirow{7}{*}{ G. subterraneus } & iDNA4mC & 0.815 & 0.822 & 0.808 & 0.630 \\
\hline & $4 \mathrm{mCPred}$ & 0.828 & 0.818 & 0.837 & 0.662 \\
\hline & 4mCPred-SVM & 0.837 & 0.840 & 0.834 & 0.674 \\
\hline & $4 \mathrm{mCCNN}$ & 0.860 & 0.851 & 0.843 & 0.703 \\
\hline & DeepTorrent & 0.880 & 0.813 & 0.948 & 0.768 \\
\hline & SOMM4mC & 0.876 & 0.864 & 0.888 & 0.728 \\
\hline & i4mC-Deep & 0.915 & 0.904 & 0.926 & 0.833 \\
\hline \multirow{7}{*}{ G. pinckeringii } & iDNA4mC & 0.831 & 0.824 & 0.838 & 0.663 \\
\hline & $4 \mathrm{mCPred}$ & 0.830 & 0.850 & 0.810 & 0.668 \\
\hline & 4mCPred-SVM & 0.860 & 0.863 & 0.858 & 0.721 \\
\hline & $4 \mathrm{mCCNN}$ & 0.871 & 0.857 & 0.893 & 0.750 \\
\hline & DeepTorrent & 0.894 & 0.831 & 0.957 & 0.795 \\
\hline & SOMM4mC & 0.903 & 0.895 & 0.911 & 0.772 \\
\hline & $\mathrm{i} 4 \mathrm{mC}$-Deep & 0.926 & 0.915 & 0.938 & 0.855 \\
\hline
\end{tabular}

Finally, we compared the proposed model with our previous published tool DNA4mCDeep [44], which was proposed for F. vesca and R. chinensis. We trained DNA4mC-Deep on the six species in this study. We found that the i4mC-Deep model performs better in 
almost all species. The comparison results of i4mC-Deep and the trained DNA4mC-Deep are given in Figure S8 and Table S1 in Supplementary File. Furthermore, we tested the pretrained cross-species model DNA4mC-Deep, and the results are given in Table S2 in Supplementary File.

C.elegans

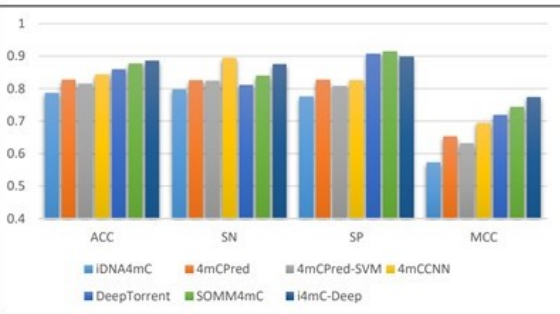

E.coli

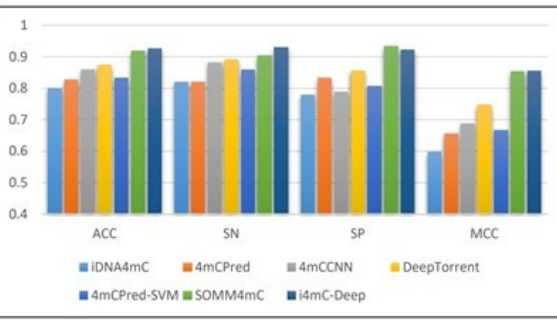

D.melanogaster

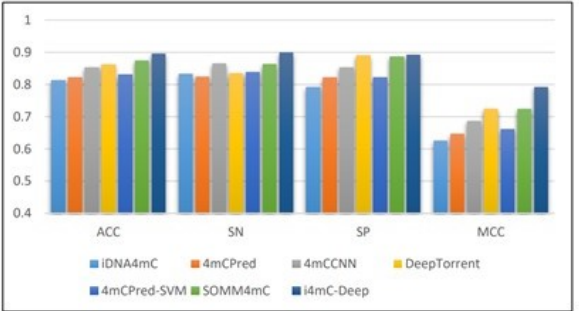

G.subterraneus

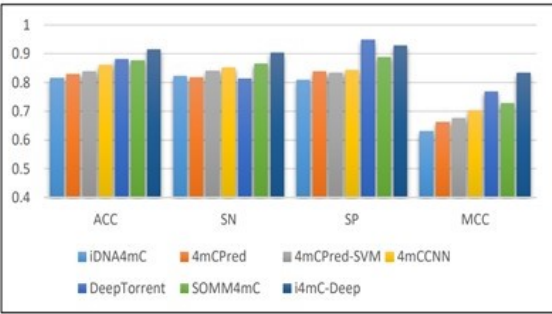

A.thaliana

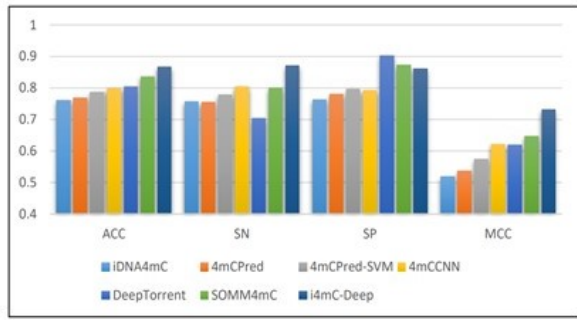

G.pickeringii

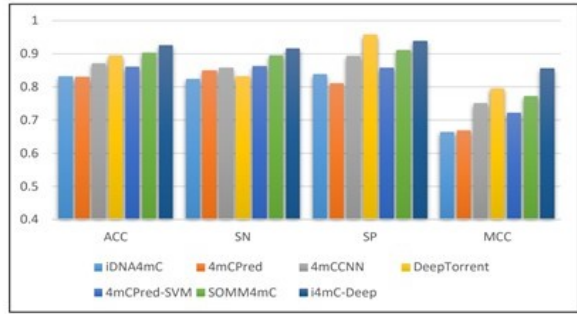

Figure 2. Shows the performance comparison of the proposed tool and other existing state-of-the-art tools.

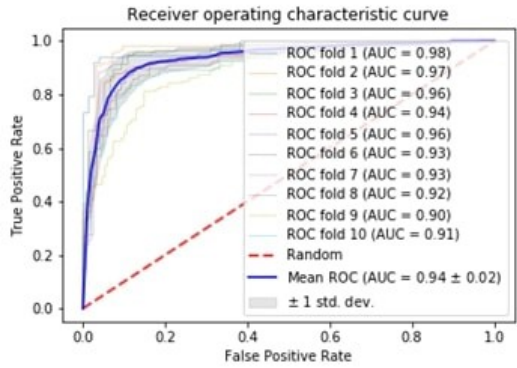

C.elegans

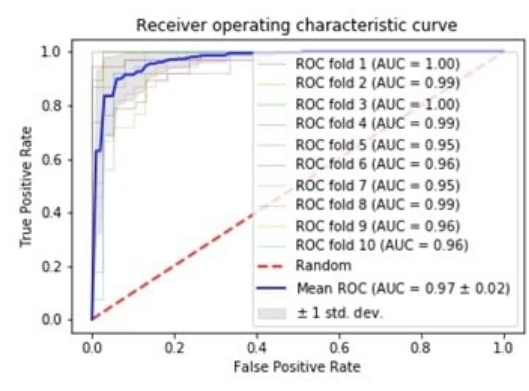

E.coli

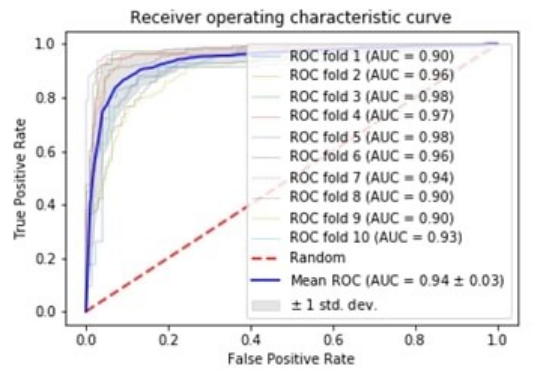

D.melanogaster

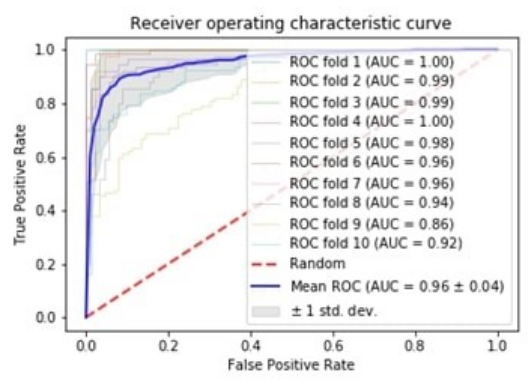

G.subterraneus

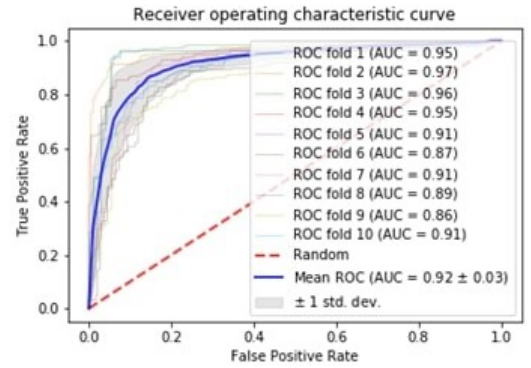

A.thaliana

Figure 3. Demonstration of the test dataset receiver operation characteristic curve (ROC) of the ten folds and their standard deviation for six species. 


\subsection{Interpretation of the Proposed Tool}

The interpretation of the trained models provides the biologist insights for a better understanding of the task at hand. The developed models for the six species learned separable features. These separable features made the task of the classifier easier and helped in outperforming the previous methods. We extracted the learned features from each trained model of every species in the study from the flatten layer. This layer represents the learned features by the model during training. Then, we used t-distributed stochastic neighbor embedding (tsne) to visualize the learned features. For example, Figure 4 shows the learned features by the G. subterraneus model. It can be seen that the proposed model was able to learn separable features so the achieved performance is superior compared with the state-of-the-art models for the same dataset. The same behavior was obtained from the other models in our study, as shown in Figure S1 in Supplementary File.

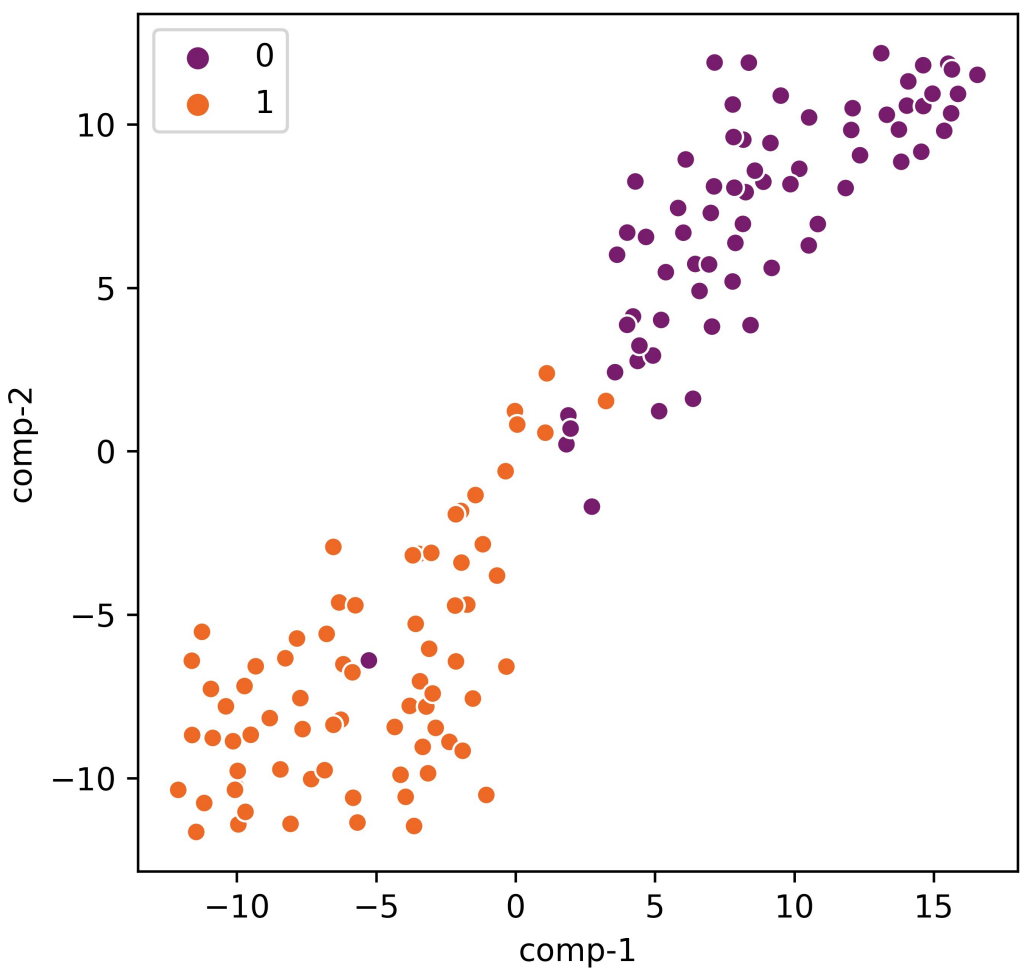

Figure 4. The t-SNE visualization of the learned features of the G. subterraneus dataset using the proposed model. The " 0 " represents the features of the negative samples and " 1 " represents the features of the positive samples.

Furthermore, we studied in silico mutagenesis using the trained models on the six species in our study. This method was applied in various studies [44-46] to interpret the effects of mutations using the trained deep learning model.

For every input sequence $s=\left(s_{0}, s_{2}, \ldots, s_{40}\right)$ we generated a $41 \times 4$ matrix by mutating each nucleotide at every position into the other nucleobase. For every in-silico mutation, we calculated the absolute prediction differences between the reference sequence and the mutated sequence.

The heat map of in silico mutation analysis is shown in Figure 5 for G. subterraneus, and Figure S2 in Supplementary File for the other species in our study. These heatmaps show that the mutation in the center of the sequence could have the highest impact on the prediction performance.

To further analyze the results, Figure 6 shows the effects of mutation on the prediction result for G. subterraneus and other species in Supplementary file Figures S3-S7. It can be seen that mutations in the flanking regions, positions 0 to 17 and positions 28 to 40 , have a 
small impact on the prediction performance. However, the mutations at positions 18 to 27 alter the prediction by more than $10 \%$. The most noticeable alteration in the prediction occurs due to the mutation to Guanine (G) at position 21 of more than $20 \%$.

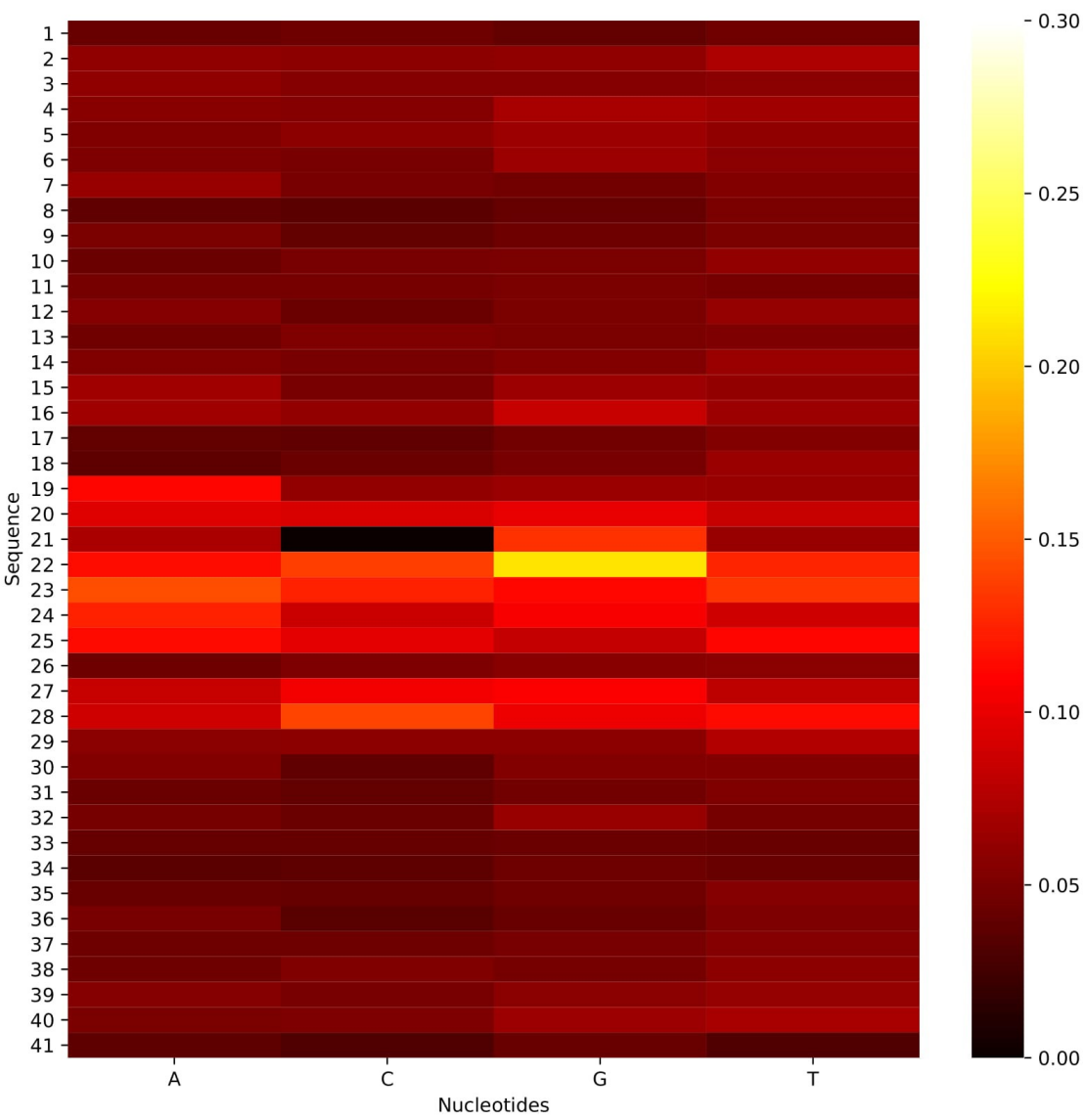

Figure 5. Demonstration of a heatmap visualization of in silico mutation of G. subterraneus.

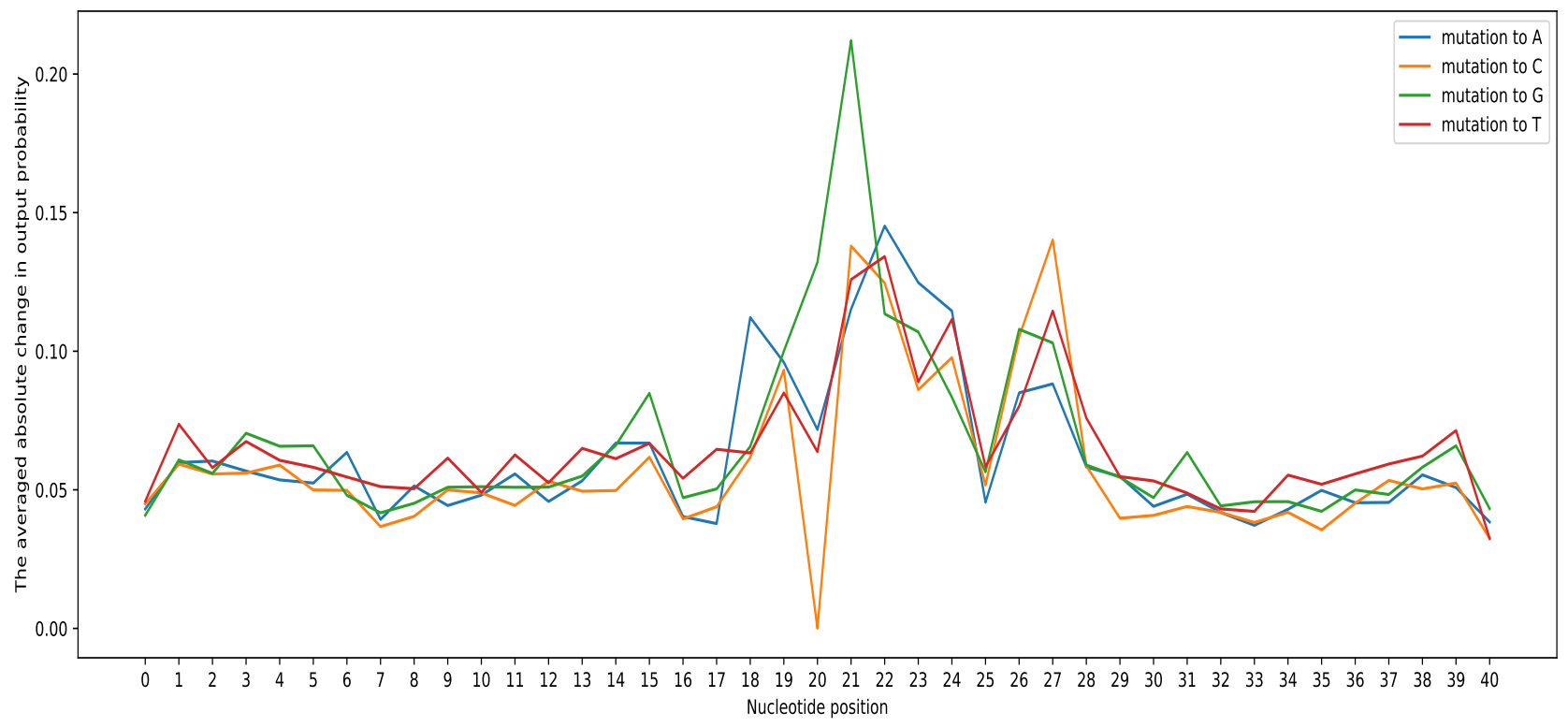

Figure 6. The effect of the mutations on the prediction probability in G. subterraneus. 


\section{Web-Server}

We established a user-friendly and freely accessible web server for the proposed method to facilitate future studies. The established web server supports the classification of $4 \mathrm{mC}$ sites using either direct sequences in Fasta format, as shown in Figure 7, or direct upload of a Fasta file, as shown in Figure 8. The web server uses the Python programming language with the Flask library. It is available at http:/ / nsclbio.jbnu.ac.kr/tools/i4mC-Deep/, accessed on 15 July 2021.

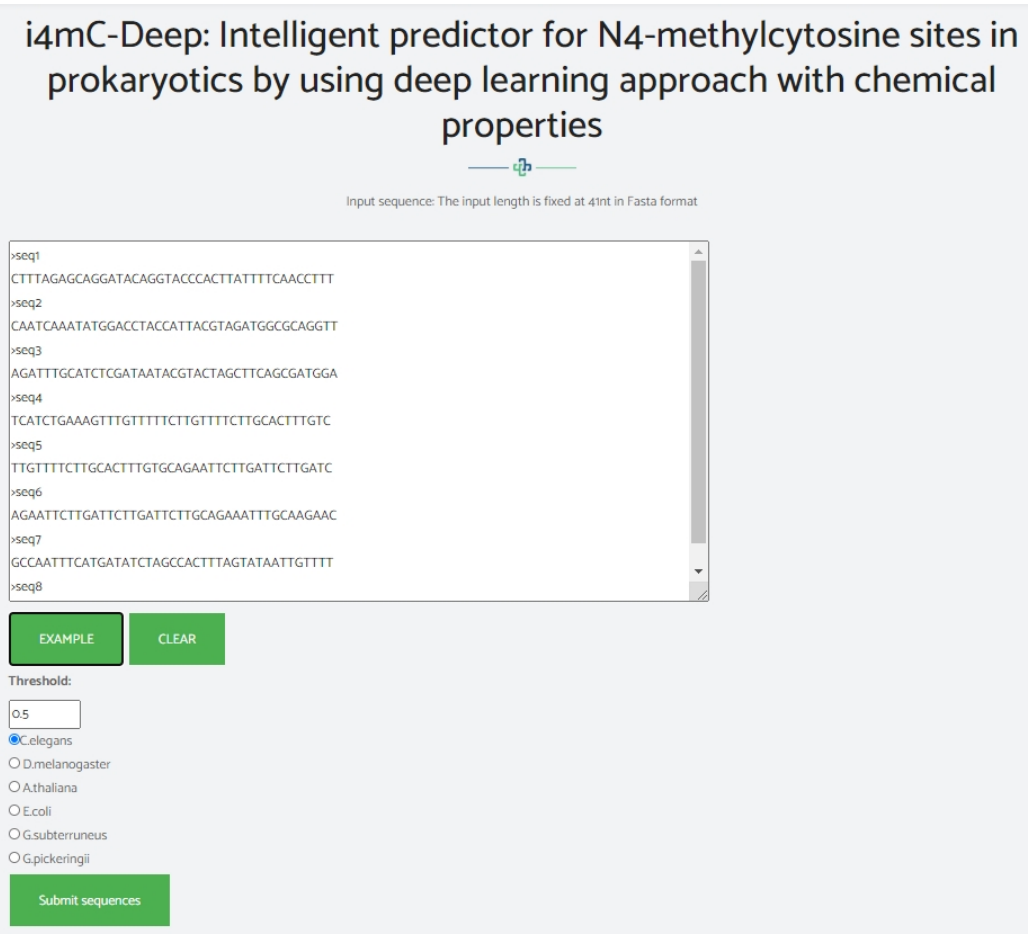

Figure 7. Demonstration of the web-server window where the users can put the DNA sequences in Fasta format directly for the prediction of $4 \mathrm{mC}$ site.

\section{Upload file}
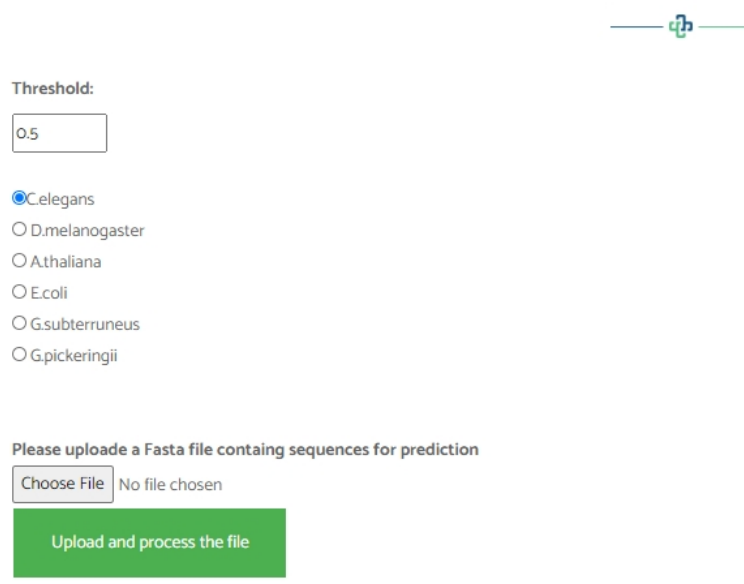

Figure 8. Demonstration of the web-server window where the users can upload the DNA sequence in the Fasta file.

\section{Conclusions}

DNA N4-methylcytosine is an important biochemical modification that regulates gene expression. Therefore, an accurate and efficient computational tool, i4mC-Deep, was developed to identify $4 \mathrm{mC}$ sites in DNA sequences. i4mC-Deep has a layered architecture 
with a convolution layer, batch normalization layer, dropout layer, and dense layer. NCP and DN techniques are used to encode a DNA sequence to discrete values. The convolution layer automatically extracts features from a given input DNA sequence. Hyper-parameter searching is applied to identify the optimal parameter. The outcomes of four evaluation metrics demonstrate that i4mC-Deep is more reliable and efficient than comparable tools. The i4mCDeep tool will be invaluable for researchers in academia and industry. Finally, we developed a web server for the proposed method, which is freely accessible online at http:/ /nsclbio.jbnu.ac.kr/tools/i4mC-Deep, accessed on 15 July 2021.

Supplementary Materials: The following are available online at https:/ / www.mdpi.com/article/10 .3390 / genes12081117/s1. Figure S1: The t-SNE visualization of the learned features for C. elegans (a), A. thaliana (b), D. melanogaster (c), E. coli (d), and G. pickeringii (e); Figure S2: Heatmaps of in silico mutagenesis analysis for C. elegans (a), A. thaliana (b), D. melanogaster (c), E. coli (d), and G. pickeringii (e); Figure S3: The effects of mutation on the prediction result in A. thaliana; Figure S4: The effects of mutation on the prediction result in C. elegans; Figure S5: The effects of mutation on the prediction result in D. melanogaster; Figure S6: The effects of mutation on the prediction result in E. coli; Figure S7: The effects of mutation on the prediction result in G. pickeringii; Figure S8: The comparison results between i4mC-Deep and DNA4mC-Deep after training the DNA4mC-Deep model on the six species in this study; Table S1: The performance comparison between the i4mC-Deep and the DNA4mCDeep after training the DNA4mC-Deep model on six species; Table S2: The performance comparison between i4mC-Deep and pretrained cross-species model DNA4mC-Deep.

Author Contributions: W.A. and H.T. prepared the dataset, conceived of the algorithm, carried out the experiments and analysis, prepared the web-server and wrote the manuscript with support from K.T.C. All authors discussed the results and contributed to the final manuscript. All authors have read and agreed to the published version of the manuscript.

Funding: This work was supported by "Human Resources Program in Energy Technology" of the Korea Institute of Energy Technology Evaluation and Planning (KETEP), granted financial resource from the Ministry of Trade, Industry and Energy, Republic of Korea. (No. 20204010600470) and the Brain Research Program of the National Research Foundation (NRF) funded by the Korean government (MSIT) (No. NRF-2017M3C7A1044816 and No. 2020R1A2C2005612).

Institutional Review Board Statement: Not applicable for this study.

Informed Consent Statement: Not applicable for this study.

Data Availability Statement: No new data were generated in this study.

Conflicts of Interest: The authors declare no conflict of interest.

\section{References}

1. Schübeler, D. Function and information content of DNA methylation. Nature 2015, 517, 321-326. [CrossRef] [PubMed]

2. Rathi, P.; Maurer, S.; Summerer, D. Selective recognition of N 4-methylcytosine in DNA by engineered transcription-activator-like effectors. Philos. Trans. R. Soc. B Biol. Sci. 2018, 373, 20170078. [CrossRef]

3. Pataillot-Meakin, T.; Pillay, N.; Beck, S. 3-methylcytosine in cancer: An underappreciated methyl lesion? Epigenomics 2016, 8, 451-454. [CrossRef] [PubMed]

4. Davis, B.M.; Chao, M.C.; Waldor, M.K. Entering the era of bacterial epigenomics with single molecule real time DNA sequencing. Curr. Opin. Microbiol. 2013, 16, 192-198. [CrossRef]

5. Gu, J.; Stevens, M.; Xing, X.; Li, D.; Zhang, B.; Payton, J.E.; Oltz, E.M.; Jarvis, J.N.; Jiang, K.; Cicero, T.; et al. Mapping of variable DNA methylation across multiple cell types defines a dynamic regulatory landscape of the human genome. G3 Genes Genomes Genet. 2016, 6, 973-986. [CrossRef]

6. Robertson, K.D. DNA methylation and human disease. Nat. Rev. Genet. 2005, 6, 597-610. [CrossRef]

7. Jones, P.A. Functions of DNA methylation: Islands, start sites, gene bodies and beyond. Nat. Rev. Genet. 2012, 13, 484-492. [CrossRef]

8. Yao, B.; Jin, P. Cytosine modifications in neurodevelopment and diseases. Cell. Mol. Life Sci. 2014, 71, 405-418. [CrossRef]

9. Ling, C.; Groop, L. Epigenetics: A molecular link between environmental factors and type 2 diabetes. Diabetes 2009, 58, 2718-2725. [CrossRef]

10. Cheng, X. DNA modification by methyltransferases. Curr. Opin. Struct. Biol. 1995, 5, 4-10. [CrossRef]

11. Chen, K.; Zhao, B.S.; He, C. Nucleic acid modifications in regulation of gene expression. Cell Chem. Biol. 2016, $23,74-85$. [CrossRef] [PubMed] 
12. Doherty, R.; Couldrey, C. Exploring genome wide bisulfite sequencing for DNA methylation analysis in livestock: A technical assessment. Front. Genet. 2014, 5, 126. [CrossRef] [PubMed]

13. Flusberg, B.A.; Webster, D.R.; Lee, J.H.; Travers, K.J.; Olivares, E.C.; Clark, T.A.; Korlach, J.; Turner, S.W. Direct detection of DNA methylation during single-molecule, real-time sequencing. Nat. Methods 2010, 7, 461. [CrossRef] [PubMed]

14. Boch, J.; Bonas, U. Xanthomonas AvrBs3 family-type III effectors: Discovery and function. Annu. Rev. Phytopathol. 2010, 48, 419-436. [CrossRef] [PubMed]

15. Ilyas, T.; Khan, A.; Umraiz, M.; Kim, H. SEEK: A Framework of Superpixel Learning with CNN Features for Unsupervised Segmentation. Electronics 2020, 9, 383. [CrossRef]

16. Khan, A.; Ilyas, T.; Umraiz, M.; Mannan, Z.I.; Kim, H. CED-Net: Crops and Weeds Segmentation for Smart Farming Using a Small Cascaded Encoder-Decoder Architecture. Electronics 2020, 9, 1602. [CrossRef]

17. Nizami, I.F.; ur Rehman, M.; Majid, M.; Anwar, S.M. Natural scene statistics model independent no-reference image quality assessment using patch based discrete cosine transform. Multimed. Tools Appl. 2020, 79, 26285-26304. [CrossRef]

18. Graves, A.; Schmidhuber, J. Framewise phoneme classification with bidirectional LSTM and other neural network architectures. Neural Networks 2005, 18, 602-610. [CrossRef]

19. Sundermeyer, M.; Alkhouli, T.; Wuebker, J.; Ney, H. Translation modeling with bidirectional recurrent neural networks. In Proceedings of the 2014 Conference on Empirical Methods in Natural Language Processing (EMNLP), Doha, Qatar, 25-29 October 2014; pp. 14-25.

20. Tayara, H.; Chong, K. Improved Predicting of The Sequence Specificities of RNA Binding Proteins by Deep Learning. IEEE Acm Trans. Comput. Biol. Bioinform. 2020. [CrossRef]

21. Rehman, M.U.; Chong, K.T. DNA6mA-MINT: DNA-6mA modification identification neural tool. Genes 2020, 11, 898. [CrossRef]

22. Tayara, H.; Tahir, M.; Chong, K.T. iSS-CNN: Identifying splicing sites using convolution neural network. Chemom. Intell. Lab. Syst. 2019, 188, 63-69. [CrossRef]

23. Alam, W.; Ali, S.D.; Tayara, H.; Chong, K.T. A CNN-Based RNA N6-Methyladenosine Site Predictor for Multiple Species Using Heterogeneous Features Representation. IEEE Access 2020, 8, 138203-138209. [CrossRef]

24. Ali, S.D.; Alam, W.; Tayara, H.; Chong, K. Identification of Functional piRNAs Using a Convolutional Neural Network. IEEE Acm Trans. Comput. Biol. Bioinform. 2020. [CrossRef] [PubMed]

25. Chen, W.; Yang, H.; Feng, P.; Ding, H.; Lin, H. iDNA4mC: Identifying DNA N4-methylcytosine sites based on nucleotide chemical properties. Bioinformatics 2017, 33, 3518-3523. [CrossRef]

26. He, W.; Jia, C.; Zou, Q. 4mCPred: Machine learning methods for DNA N4-methylcytosine sites prediction. Bioinformatics 2019, 35, 593-601. [CrossRef] [PubMed]

27. Wei, L.; Luan, S.; Nagai, L.A.E.; Su, R.; Zou, Q. Exploring sequence-based features for the improved prediction of DNA N4-methylcytosine sites in multiple species. Bioinformatics 2019, 35, 1326-1333. [CrossRef] [PubMed]

28. Yang, J.; Lang, K.; Zhang, G.; Fan, X.; Chen, Y.; Pian, C. SOMM4mC: A second-order Markov model for DNA N4-methylcytosine site prediction in six species. Bioinformatics 2020, 36, 4103-4105. [CrossRef] [PubMed]

29. Khanal, J.; Nazari, I.; Tayara, H.; Chong, K.T. 4mCCNN: Identification of N4-methylcytosine sites in prokaryotes using convolutional neural network. IEEE Access 2019, 7, 145455-145461. [CrossRef]

30. Liu, Q.; Chen, J.; Wang, Y.; Li, S.; Jia, C.; Song, J.; Li, F. DeepTorrent: A deep learning-based approach for predicting DNA N4-methylcytosine sites. Briefings Bioinform. 2020, 22, bbaa124. [CrossRef]

31. Bari, A.G.; Reaz, M.R.; Choi, H.J.; Jeong, B.S. DNA encoding for splice site prediction in large DNA sequence. In Proceedings of the International Conference on Database Systems for Advanced Applications; Springer: Berlin/Heidelberg, Germany, 2013 ; pp. 46-58.

32. Alam, W.; Tayara, H.; Chong, K.T. XG-ac4C: Identification of N4-acetylcytidine (ac4C) in mRNA using eXtreme gradient boosting with electron-ion interaction pseudopotentials. Sci. Rep. 2020, 10, 20942. [CrossRef]

33. Tahir, M.; Hayat, M. iNuc-STNC: A sequence-based predictor for identification of nucleosome positioning in genomes by extending the concept of SAAC and Chou's PseAAC. Mol. BioSystems 2016, 12, 2587-2593. [CrossRef]

34. Tahir, M.; Hayat, M. Machine learning based identification of protein-protein interactions using derived features of physiochemical properties and evolutionary profiles. Artif. Intell. Med. 2017, 78, 61-71. [CrossRef]

35. Tahir, M.; Hayat, M.; Kabir, M. Sequence based predictor for discrimination of enhancer and their types by applying general form of Chou's trinucleotide composition. Comput. Methods Programs Biomed. 2017, 146, 69-75. [CrossRef]

36. Ye, P.; Luan, Y.; Chen, K.; Liu, Y.; Xiao, C.; Xie, Z. MethSMRT: An integrative database for DNA N6-methyladenine and N4-methylcytosine generated by single-molecular real-time sequencing. Nucleic Acids Res. 2016, 45, gkw950. [CrossRef]

37. Wahab, A.; Ali, S.D.; Tayara, H.; Chong, K.T. iIM-CNN: Intelligent identifier of $6 \mathrm{~mA}$ sites on different species by using convolution neural network. IEEE Access 2019, 7, 178577-178583. [CrossRef]

38. Chantsalnyam, T.; Lim, D.Y.; Tayara, H.; Chong, K.T. ncRDeep: Non-coding RNA classification with convolutional neural network. Comput. Biol. Chem. 2020, 88, 107364. [CrossRef] [PubMed]

39. Ali, S.D.; Kim, J.H.; Tayara, H.; Chong, K.T. Prediction of RNA 5-Hydroxymethylcytosine Modifications Using Deep Learning. IEEE Access 2021, 9, 8491-8496. [CrossRef]

40. Siraj, A.; Lim, D.Y.; Tayara, H.; Chong, K.T. UbiComb: A Hybrid Deep Learning Model for Predicting Plant-Specific Protein Ubiquitylation Sites. Genes 2021, 12, 717. [CrossRef] 
41. Tahir, M.; Tayara, H.; Chong, K.T. iDNA6mA (5-step rule): Identification of DNA N6-methyladenine sites in the rice genome by intelligent computational model via Chou's 5-step rule. Chemom. Intell. Lab. Syst. 2019, 189, 96-101. [CrossRef]

42. Tahir, M.; Hayat, M.; Ullah, I.; Chong, K.T. A deep learning-based computational approach for discrimination of DNA N6methyladenosine sites by fusing heterogeneous features. Chemom. Intell. Lab. Syst. 2020, 206, 104151. [CrossRef]

43. Siraj, A.; Chantsalnyam, T.; Tayara, H.; Chong, K.T. Recsno: Prediction of protein s-nitrosylation sites using a recurrent neural network. IEEE Access 2021, 9, 6674-6682. [CrossRef]

44. Wahab, A.; Mahmoudi, O.; Kim, J.; Chong, K.T. DNC4mC-Deep: Identification and analysis of DNA N4-methylcytosine sites based on different encoding schemes by using deep learning. Cells 2020, 9, 1756. [CrossRef] [PubMed]

45. Zou, J.; Huss, M.; Abid, A.; Mohammadi, P.; Torkamani, A.; Telenti, A. A primer on deep learning in genomics. Nat. Genet. 2019, 51, 12-18. [CrossRef] [PubMed]

46. Raimondi, D.; Orlando, G.; Tabaro, F.; Lenaerts, T.; Rooman, M.; Moreau, Y.; Vranken, W.F. Large-scale in-silico statistical mutagenesis analysis sheds light on the deleteriousness landscape of the human proteome. Sci. Rep. 2018, 8, 16980. [CrossRef] [PubMed] 